\title{
Colonic diverticula
}

\author{
Martin Eastwood \\ Gastrointestinal Unit, Department of Medical Sciences, University of Edinburgh, Western General Hospital, \\ Edinburgh EH4 $2 X U$, UK
}

\begin{abstract}
Diverticulosis is a condition that is associated with ageing. The older the individual the more likely they are to have diverticulosis, but this process is not necessarily inevitable. Diverticula are a herniation through the wall of the sigmoid colon and are likely to be a consequence of a weakness in the colonic wall or prolonged exposure to increased intracolonic pressure consequent on a low dietary fibre intake. The tensile strength of the colon declines with age and becomes least in the distal colon. Adult Africans living in Africa eat a high-fibre diet and are free from diverticulosis. Their colons are stronger, wider and thinner than those Scottish adults of the same age. The strength of the colon is in part dependent on collagen fibres and their diameter. The collagen fibrils on the left side of the colon are smaller and more tightly packed than those of the right side of the colon. Ageing and the presence of colonic diverticulosis are associated with smaller more-tightlypacked collagen fibrils. An animal model was established to investigate the effect of a lifelong high- and low-fibre diet on the development of colonic diverticulosis. The findings show that a high-fibre diet from birth, and preferably a maternal high-fibre diet, lessen the risk of diverticulosis with age.
\end{abstract}

Colonic diverticulosis: Colon structure: Collagen fibrils: Dietary fibre

\section{The colon, anatomy and function}

The human colon is approximately $1.5 \mathrm{~m}$ long and arches from the right iliac fossa up to the liver, across to the left hypochondrium and descends along the left side of the abdomen to the anus. The 'colon' consists of the caecum, colon (ascending, transverse, descending and sigmoid), rectum and anal canal, and is suspended from a mesentery in the transverse and sigmoid colon. The layers of the colon are made up of the mucosa (epithelium, lamina propria and the muscularis mucosae), the submucosa and the muscularis propria. The epithelium of the mucosa lining the colon is glandular and the principal cell type is a columnar absorptive cell. The lamina propria and the submucosa contain strands of collagen. The main smooth muscles of the colon form two layers: the outer longitudinal muscle layer, which is thickened to form three taenia; the inner circular muscle layer, which is circumferential and is thickened in the anal canal to form the rectum (Christensen, 1991).

The colon can also be regarded in terms of function as two organs. The right side of the colon is a fermenter and absorptive organ, and the left side is a regulator of faecal output. By the end of the fermentation process the considerable absorptive capacity of the right side reduces the large volumes of fluid flowing from the ileum to faeces. Approximately 1-21 ileal effluent/d are converted to the 'plasticine'-like faeces, which range in weight from 25 to $300 \mathrm{~g}$. On the left side of the colon continence and the passage of faeces is controlled by the contraction of the circular and longitudinal muscles (Eastwood, 1998).

\section{Colonic diverticulosis}

Colonic diverticulosis of the left, especially sigmoid, colon is a common condition in Western countries. This condition is in general benign, being rare before the age of 30 years and increasing in incidence with age. In the UK population one-third will develop colonic diverticulosis by the sixth decade and half by the ninth decade. There are differences in incidence in the UK, the number of cases admitted to hospital in Scotland is approximately double that of England and Wales (range 20-66 per 100000 of the population). Within Scotland the rate in the north is double that of Scotland as a whole. Similar differences occur in different Scandinavian countries (Eastwood, 1987). 


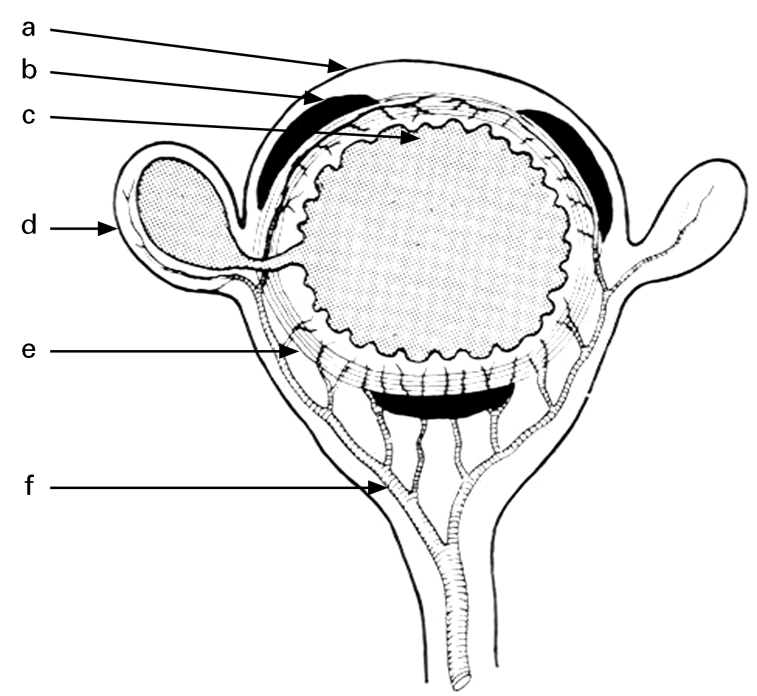

Fig. 1. Diagram of cross section of the human colon illustrating the change in structure associated with colonic diverticulosis. a, Colon wall; b, c, mucosa; d, diverticulum, e, muscle; f, blood vessels. (From Drummond, 1917.)

Colonic diverticulosis occurs on the underside of the colon, with two rows of diverticula between the mesenteric and medial and lateral antimesenteric taeniae (Fig. 1). In $50 \%$ of the advanced cases a third row of diverticula is found between the two antimesenteric taeniae (Shepherd, 1992). The changes in the colonic wall are characterised by a thickening of the colonic muscle and pulsion diverticula. The diverticula consist of a pouch of mucus membrane projecting through the circular muscle layers to the pericolic fat and appendices epiploicae. The main blood vessels supply the colon from the mesentery, which holds the colon in place. Where these vessels penetrate the muscle wall they form a tunnel, which is a point of weakness that facilitates diverticula formation.

Most individuals with colonic diverticulosis are unaware of the structural changes in the colonic musculature and wall. Some individuals, however, experience various extents of clinically significant complications of diverticulosis. Symptomatic diverticular disease (sometimes wrongly termed diverticulitis) presents with left-sided abdominal pain relieved by the passage of small pellet-like stools. This pain is secondary to increased pressure in the sigmoid colon. Less frequently, diverticulosis can be complicated by true inflammation, fistula to other organs or haemorrhage (Eastwood, 1987).

\section{Hypotheses on the aetiology of colonic diverticulosis}

In the 1970s Neil Painter (1975) proposed that a deficiency of dietary fibre is the cause of diverticular disease. Painter's (1975) hypothesis was that high pressures in the colon and prolonged gastrointestinal transit time were a consequence of a fibre-deficient diet. He argued that the circular muscles of the left side and sigmoid colon need to contract strongly in order to transmit and expel the small stool associated with a fibre-deficient diet. Years of squeezing such inspissated stools by increased pressure within the segmented section of

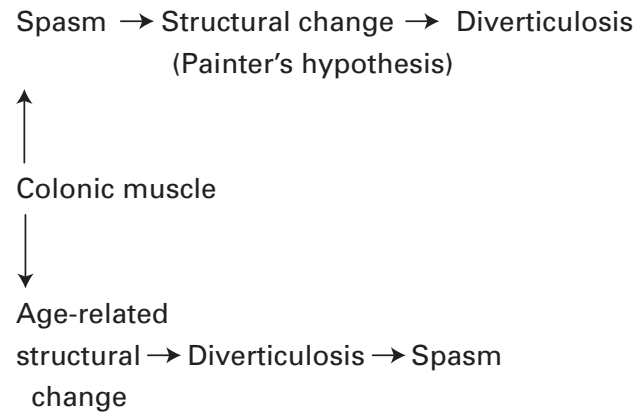

Fig. 2. The two theories for the aetiology of colonic diverticulosis.

bowel leads to diverticulosis (Fig. 2). The African, having a large dietary fibre intake and regularly passing a large stool, is not liable to this problem. The differences in prevalence of diverticular disease in England, Wales and Scotland and between the regions of Scotland could, to some extent, parallel differences in fruit and vegetable intake.

\section{Clinical features of individuals with colonic diverticulosis}

Painter's (1975) concept was an attractive one. As a consequence, patients presenting to a gastrointestinal unit in Edinburgh who had been shown by barium enema to have colonic diverticulosis were investigated. Stool weight (3d), trans-intestinal transit time and colonic motility were measured. The range of stool weight in a $24 \mathrm{~h}$ period and trans-intestinal transit times in these individuals was indistinguishable from values obtained for a normal local population of similar age distribution. Fasting- and foodstimulated motility and pressure measurements in the sigmoid colon gave both basal and stimulated motility indices extending across a wide range of values, the majority of which were low. It is only when spasm develops that symptoms develop in the otherwise benign condition of diverticulosis (Royal College of Physicians, 1980).

In a number of studies we supplemented the diet with coarse wheat bran (range of increment $12-39 \mathrm{~g} / \mathrm{d}$ ) for symptom-free volunteers not receiving health care and patients with symptomatic colonic diverticulosis, irritable bowel and simple constipation. The effect on stool weight of supplementing the diet with bran was different in these groups. In the symptom-free volunteers the range of increase in faecal weight as a result of taking bran ranged from 1.9 to $5.4 \mathrm{~g}$ wet faecal weight/g wheat bran. Similar results were obtained for individuals who were constipated (about $2 \mathrm{~g}$ wet faecal weight/g wheat bran). However, for patients with irritable bowel syndrome and symptomatic diverticulosis the response to wheat bran was between 0.9 and $1.9 \mathrm{~g}$ wet faecal weight/g wheat bran. This finding implies that where there is spasm in the colon the response to bran was reduced (Eastwood, 1998).

There are two possible pathways by which colonic diverticulosis could develop (Fig. 2): (1) a diet deficient in 
fibre results in a small faecal output and increased pressure in the colon. Such pressure forces the colonic mucosa to protrude through the wall at the point where the main blood vessel penetrates the muscle wall and as a result of this structural change, diverticulosis develops (Painter, 1975); (2) there is weakening of the colonic wall with age, allowing protrusions and the formation of diverticula in the descending and sigmoid colon.

\section{Colonic wall strength and development of colonic diverticulosis}

\section{Mechanical properties}

Iwasaki (cited by Yamade, 1970) found that the tensile strength of the colon obtained post mortem in Japan declined with age. Taking this observation further, the possibility of a change in the physical properties of the bowel wall was investigated in patients with diverticular disease. Burkitt (Burkitt \& Trowell, 1975) suggested that the mechanical properties of the colon might be different in African and the European subjects. The reason was that Africans, in whom diverticular disease is reputedly rare, eat a high-fibre diet that results in fast intestinal transit times and bulky stools (Cleave, 1974).

The mechanical properties of samples of colon obtained post mortem in Edinburgh, Scotland and Kampala, Uganda were investigated in order to determine whether these properties were uniform throughout the length of the colon, whether they changed with age and whether there were any possible changes predisposing to the development of diverticulosis (Watters et al. 1985). Mechanical properties were tested using an Instron 1026 tensiometer (Instrom Corp., Canton, MA, USA). The following measurements were made: width, thickness and internal diameter of the bowel, burst strength, tensile strength, thickness, internal diameter, width at burst, percentage elongation, stress and relaxation. Burst strength $(\mathrm{g})$ is the force required to disrupt the tissue ring, being a measure of the strength of the tissue components in the colon wall, predominantly collagen. The tensile strength is derived from the burst strength and thickness of the colonic wall, being a measure of the stress per unit area and expressed as $\mathrm{g} / \mathrm{mm}^{2}$ cross-sectional area of colon.

The burst strength did not differ across the colon. Burst and tensile strength decreased with age throughout the colon. There was a tendency for the cross-sectional area and thickness of the colon to increase with age but this trend was not significant. The thickness of the colon was less in the Kampala group, but the difference was only significant $(P \geq 0.05)$ in the sigmoid colon. The internal diameter of the colon decreased with age and the distal colon in the Kampala group was wider than that in the Edinburgh group (descending colon $P \geq 0 \cdot 05$ ). There was a trend in the Kampala group for the colon to be stronger, wider and thinner, thus being more mechanically efficient. The ability of the colon to stretch was measured by the width at burst, and remained constant with age in the proximal colon without any difference between the two groups. The distal colon showed a marked tendency with age to a diminished width at burst $(P<0.001)$, but the Kampala subjects had a significantly greater stretch capacity in the distal colon (descending $P<0 \cdot 01$, sigmoid $P<0 \cdot 001$ ) when compared with the colons of the Edinburgh subjects. Visco-elastic properties were similar for both groups and showed no tendency to change with age. The distal colon in both groups had a steeper stress relaxation slope than the proximal colon.

In the Edinburgh group individuals with diverticular disease did not differ from individuals aged $>50$ years with a normal colon for any of the measurements made (tensile strength, internal diameter and width at burst).

These results are in accordance with the findings of Iwasaki (cited by Yamade, 1970), whose values for tensile strength were similar to those in the present study for each age and site studied. The greater tensile strength in the Kampala group was related to an increase in burst strength and reduced thickness. It appears that the fall in tensile strength with age is not therefore a measure of increasing thickness, but rather due to a decrease in the integrity of the connective tissue. It would appear, therefore, that the distal colon is weaker and less expansile in old age and also narrower, weaker and less expansile than the proximal colon. This finding is also consistent with the fact that the distal colon has been shown to be most prone to develop colonic diverticulosis in populations of Western countries. Patients with colonic diverticulosis had similar mechanical properties to those without colonic diverticulosis. The similarity between the two groups suggests that the changes that have occurred in the distal colon with age make it more vulnerable to colonic diverticulosis rather than the mechanical changes occurring as a result of diverticulosis (Watters et al. 1985).

\section{Colon structure}

The submucosa of the colon is the layer that is composed almost entirely of collagen and is resistant to shearing forces (Halsted, 1891; Epstein \& Munderloh, 1975). Connective tissue contributes a substantial proportion of the strength of the colonic wall. The mechanical properties of connective tissue depend on a wide variety of factors: the type of tissue and its age; the nature of the intramolecular and intermolecular covalent cross-links; the quantity of the glycosaminoglycans associated with the collagen fibrils (Parry et al. 1978; Flint et al. 1984). Collagen is arranged in a criss-cross fishnet band and is combined with a readilyexpansible matrix of ground substance and elastic tissue. As the colonic wall is stretched, the initial slack collagen fibres take up the stress and eventually become taut. The distensibility of the colon is limited by the angle between individual collagen fibres, which is more acute from the serosal to the mucosal side. The mucosal layer is possibly more elastic, and it is likely that the stiffer external layers break and allow the elastic mucosa to herniate through to form a diverticulum.

In further studies the colonic collagen was examined using the dissection method developed by Ferraz de Carvalho (1973). The collagen fibril diameter increases from birth to maturity and decreases thereafter throughout adult life. The mechanical properties and tensile strength of a connective tissue are positively related to the diameter of 
the constituent collagen fibrils; large collagen fibrils having greater tensile strength than small fibrils (Parry et al. 1978; Flint et al. 1984). A reduction in the diameter of the collagen fibrils will increase the interfibrillar non-covalent crosslinks between the collagen fibrils and the matrix components. This process increases the density of intrafibrillar covalent cross-linkages between the collagen fibrils and the matrix components (Flint et al. 1984). The submucosal collagen is arranged in layers $(0 \cdot 5-2 \cdot 0 \mu \mathrm{m}$ in thickness) and forms a network orientated in two axes, a stratified structure with all the collagen fibrils in each layer running in the same direction. The fibrils range in diameter from 4 to $104 \mathrm{~nm}$. The mean fibril diameter is 69 (SD 13) nm and mean fibril count is 159 (SD 58)/ $/ \mathrm{m}^{2}$ (Thomson et al. 1986).

There was a significant tendency for the fibrils to be smaller and more tightly packed on the left side of the colon compared with the right (diameters $66 \mathrm{~nm} \mathrm{(SD} \mathrm{11)} \mathrm{and}$ $78 \mathrm{~nm}$ (SD 11) respectively; $P<0.001$ ) and fibril count to be greater on the left side than on the right side (58 (SD 14), 46 (SD 13)/print field respectively; $P<0 \cdot 001$ ). There was no significant difference between gender for fibril diameter count between the two sides of the colon. Ageing is associated with smaller, tighter-packed fibrils, and fibril diameter and fibril count for the left side of the colon were related to age. In individuals with colonic diverticulosis the fibril count for the left side of the colon was higher $(P<0.05)$ and fibril diameters were lower than those for colons of comparable age without diverticula. The changes normally associated with ageing are more pronounced in colonic diverticulosis and may represent a premature ageing process (Thomson et al. 1987).

\section{Collagen structure}

The collagen in the colon is both type I and type III (Epstein \& Munderloh, 1975). Intermolecular cross-links give stability to the collagen-containing tissue by stabilising the molecular arrangement within collagen fibrils (Bornstein \& Pieze, 1966). Cross-linking in collagen is based on aldehyde formation by lysine or hydroxylysine residues. Collagen solubility in weak acids is indirectly related to the extent of cross-linkage in the collagen of the tissue in the study. The insoluble:soluble collagen value (the solubility index) gives an indication of the amount and possibly the nature of crosslinks present. A higher solubility index indicates a higher extent of cross-linkage of the collagen molecules. Acidsoluble collagen contains aldimine cross-links (-CH2-CN-). The insoluble residues form ketoimine (-CO-CH2-NA-) cross-links, mature cross-links of both the allysine and hydroxyallysine pathways and advanced glycation products. Accumulation of covalently-linked sugar molecules and related increased cross-linking products are found in a variety of tissues with ageing, including skin, vascular, adventitia and the cordae tendinae of heart valves.

\section{Studies in man}

Collagen and the cross-linkage of that collagen were measured in tissue obtained from colons free from, or with, colonic diverticulosis. The total overall collagen content of these human colons was $158 \mathrm{mg} / \mathrm{g}$ wet weight of colon, and did not vary between the left and right side (Wess et al. 1995). There was, however, a steady increase in the proportion of insoluble collagen with age, particularly after 40 years of age. The mean values for the solubility index for control subjects $<60$ years, control subjects $>60$ years and subjects $>60$ years with diverticulosis in the sigmoid colon were 5.4 (SD 1.7); 10.3 (SD 2.5) and 17.1 (SD 2.8) respectively. These differences were highly significant $(P<0.001)$. If the extent of collagen cross-linkage increases, the tissue becomes more rigid and thus suggests that there are enhanced cross-linkages and hence less elastic walls with age and particularly in colonic diverticulosis.

\section{Rat model}

The relationship between collagen properties and age were investigated in a rat model, using male Wistar rats (Wess et al. 1996b). Previous studies have shown that rabbits and rats fed a low-fibre high-fat diet develop colonic diverticula (Hodgson, 1972). The rats, bred specifically for this study, were fed either a low-fibre diet $(17 \mathrm{~g} \mathrm{NSP} / \mathrm{kg})$ or a high-fibre $\operatorname{diet}(133 \mathrm{~g} \mathrm{NSP} / 100 \mathrm{~g}$ ) for 18 months. The diets were isoenergetic and differed not only in dietary fibre content but also in protein and utilisable carbohydrate. The percentage difference between the two diets was protein 50 and utilisable carbohydrate 27 (g/kg dry weight; protein: fibredeficient diet 178, high-fibre diet 205; utilisable carbohydrate: fibre-deficient diet 730, high-fibre diet 575). The animals were killed at 18 months and subjected to a postmortem by a consultant pathologist who was not aware of the diet eaten by the animals and was looking in particular for diverticula in the colon. Caecal and colonic contents were weighed and levels of short-chain fatty acids measured. The total collagen (based on hydroxyproline content) and collagen solubility in weak acid (acid solubility) was measured to assess the extent of cross-linkage. In the fibre-deficient group $41 \%$ had colonic diverticula compared with no diverticula in the high-fibre group. The diverticula were multiple in all instances and found in all regions in the transverse and descending colon. The total collagen content was similar in both groups and not affected by the presence of the colonic diverticulosis. The acid-solubility indices were significantly lower for the fibre-deficient group than for the lower fibre group for all sections of the large bowel $(P<0 \cdot 001)$.

Those rats in the fibre-deficient group with colonic diverticulosis had significantly lower solubility indices than those without diverticulosis $(P<0 \cdot 1)$. There was a decrease in the acid solubility index in the left side of the colon from the fibre-deficient group, but not in that from the high-fibre group. The solubility index in the ascending colons from rats fed the fibre-deficient diet that did not have diverticulosis was significantly different from that in sigmoid colons from rats fed the fibre-deficient diet that had colonic diverticulosis $(P<0 \cdot 001)$.

At 18 months the rats fed the high-fibre diet showed a $70 \%$ increase in colonic contents and the caecal short-chain fatty acid concentrations were increased $>5$-fold. The change in the physical properties of the colonic contents and the additional force necessary to propel them may have had some effect on the development of cross-links. 


\section{Influence of maternal diet}

In designing the experiment there was a problem in deciding at what stage the young rats could start on a high-fibre or low-fibre diet. Consequently, the effect of maternal diet and weaning on the development of diverticulosis with highand low-fibre diets was investigated (Wess et al. 1996a). In addition, maternal diet may have an effect on the health of the offspring in middle and later life, as has been suggested by Barker et al. (1993). The animals were divided into three groups: (1) low-fibre diet-fed young bred from a low-fibre diet-fed mother; (2) low-fibre diet-fed young bred from a high-fibre diet-fed mother; (3) control groups, i.e. high-fibre diet-fed young bred from a high-fibre diet-fed mother.

The mother was fed on the test diet for 1 month before the pregnancy. Each group of baby rats was weaned onto one of the two diets after $17 \mathrm{~d}$ suckling and they remained on that diet thereafter. Animals were killed at 18 months and subjected to a postmortem. The percentage of animals with colonic diverticulosis in the control group (high-fibre diet-fed young bred from a high-fibre diet-fed mother) was 0 . In the low-fibre diet-fed young bred from the high-fibre diet-fed mother the incidence of colonic diverticulosis was $21 \%$ and in the low-fibre diet-fed young bred from the lowfibre diet-fed mother it was $42 \%$. All the diverticula were found in the mid and left side of the colon, as in human subjects.

The total collagen content $(\mathrm{mg} / \mathrm{g}$ wet tissue colonic weight) was similar throughout the colon for all feeding regimens regardless of the presence or absence of colonic diverticulosis. The collagen acid-solubility index of the control group (high-fibre diet-fed young bred from highfibre diet-fed mothers) was consistent throughout the colon. The acid-solubility index was lower in the sigmoid colon compared with the ascending colon in the two low-fibre diet-fed offspring groups $(P \geq 0 \cdot 01)$. The change was less for the low-fibre diet-fed young bred from a hig-fibre diet-fed mother. The acid solubility index was lower in those rats with colonic diverticulosis compared with their healthy counterparts for all sides of the colon.

It is difficult to explain the difference between these groups and the effect of the maternal diet. It is possible that maternal diet may affect the colonic environment by changing the physiology of the colon. It may be that some substance or agent in the milk of high-fibre diet-fed mothers may affect the gut flora in the colon. It is possible that the bacterial content of the colon in the three different groups of animals is different, with the low-fibre diet-fed young bred from low-fibre diet-fed mothers being quite different from that of the control group (high-fibre diet-fed young bred from high-fibre diet-fed mothers), with the third group being intermediate between these two groups. Another possible explanation is that there is a dietary deficiency in the low-fibre diet-fed young bred from low-fibre diet-fed mothers, possibly involving vitamins and trace metals (e.g. vitamin $\mathrm{C}, \mathrm{Cu}$ or $\mathrm{Si}$; Tsuchiya \& Bates, 1997). These substances are cofactors in the enzymes involved in collagen synthesis. There may be differences in growth hormone levels in the milk of fibre-deficient mothers. These explanations are, however, speculative. Overall, the findings are in keeping with those of Barker et al. (1993) that the maternal diet is important in the lifelong health of an individual.

\section{References}

Barker DJE, Gluckman PD, Godfrey KM, Harding JE, Owens JA \& Robinson JS (1993) Foetal nutrition and cardiovascular disease in the adult life. Lancet 341, 938-941.

Bornstein P \& Pieze KA (1966) The nature of the intramolecular cross links in collagen. The separation and characterisation of peptides from the cross link region rat skin collagen. Biochemistry 5, 3460-3473.

Burkitt DP \& Trowell HC, editors (1975) Refined Carbohydrate Foods and Disease. Some Implications of Dietary Fibre. London: Academic Press.

Christensen J (1991) Gross and microscopic anatomy of the large intestine. In The Large Intestine Physiology, Pathophysiology and Disease, pp. 13-35 [SF Phillips, JH Pemberton and RG Shorter, editors]. New York: Raven Press.

Cleave TL (1974) The Saccharine Disease. Bristol: Wright.

Drummond H (1917) Sacculi of the large intestine with special reference to their relation to the blood vessels of the bowel wall. British Journal of Surgery 4, 407.

Eastwood MA (1987) Diverticular disease. In Oxford Textbook of Medicine, 2nd ed., pp. 12.133-12.137 [DJ Weatherall, JGG Ledingham and DA Warrell, editors]. Oxford: Oxford University Press.

Eastwood MA (1998) Structure and function of the colon. In Encyclopedia of Human Nutrition, pp. 945-953 [MJ Sadler, JJ Strain and B Caballero, editors]. San Diego, CA: Academic Press.

Epstein EH \& Munderloh NH (1975) Isolation and characterization of $\mathrm{CNBr}$ peptides of human alpha 1 (III) 3 collagen and tissue distribution of alpha 1 (I) 2 alpha 2 and alpha 1 (III) 2 alpha 3 collagen. Journal of Biological Chemistry 250, 9304-9312.

Ferraz de Carvalho CA (1973) Contribution to the functional study of tubular viscera. Arquivos de Gastroenterologia 10, 183-190.

Flint MH, Craig AS, Riley HC, Gillard GC \& Parry DAD (1984) Collagen fibril diameters and glycosaminoglycan content of skins - indices of tissue maturity and function. Connective Tissue Research 13, 69-81.

Halsted WS (1891) Intestinal anastamosis. Bulletin of Johns Hopkins Hospital 2, 1-4.

Hodgson J (1972) An animal model to study diverticular disease. British Journal of Surgery 59, 315-318.

Painter NS (1975) Diverticular Disease of the Colon. London: William Heinemann Medical Books Ltd.

Parry DAD, Barnes GRG \& Craig AS (1978) A comparison of the size distribution of collagen fibrils in connective tissues as a function of age and a possible relation between fibril size distribution and mechanical properties. Proceedings of the Royal Society of London 203B, 305-321.

Royal College of Physicians (1980) Medical Aspects of Dietary Fibre, pp. 111-117. London: Pitman Medical.

Shepherd NA (1992) Diverticular disease. In Oxford Textbook of Pathology, pp. 1256-1258 [J O'D McGee, PG Isaacson and NA Wright, editors]. Oxford: Oxford University Press.

Thomson HJ, Busuttil A, Eastwood MA, Smith AN \& Dalton RA (1986) Submucosa of the human colon. Journal of Ultrastructure and Molecular Structure Research 96, 22-30.

Thomson HG, Busuttil A, Eastwood MA, Smith AN \& Elton RA (1987) Submucosal collagen changes in the normal colon and in diverticular disease. International Journal of Colorectal Disease 20, 208-213. 
Tsuchiya H \& Bates CJ (1997) Vitamin C and copper interactions in guinea pigs in a study of collagen cross links. British Journal of Nutrition 77, 315-325.

Watters DAK, Smith AN, Eastwood MA, Anderson KC, Elton RA \& Mugerwa JW (1985) Mechanical properties of the colon: comparison of the features of the African and European colon in vitro. Gut 26, 384-392.

Wess L, Eastwood M, Busuttil A, Edwards C \& Millar A (1996a) An association between maternal diet and colonic diverticulosis in an animal model. Gut 39, 423-427.
Wess L, Eastwood MA, Edwards CA, Busuttil A \& Miller A (1996b) Collagen alterations in an animal model of colonic diverticulosis. Gut 38, 701-706.

Wess L, Eastwood MA, Wess TJ, Busuttil A \& Miller A (1995) Cross linkage of collagen is increased in colonic diverticulosis. Gut 37, 91-94.

Yamade MABA (1970) Strength of Biological Materials. Baltimore, MD: Williams and Watkins. 